in communication skills to provide basic health and family welfare education to the masses at large. The village health guides scheme needs to be rejuvenated and made functional. It is necessary during the VIIIth plan to create demand generation for health and family welfare particularly family wel- fare schemes. The public must demand family welfare services like they demand ration, atta or sugar. The health and family welfare programme must become a people's movement without which the national development itself is getting confined.

\title{
SUDDEN RESPIRATORY ARREST IN ASTHMA
}

The rates of mortality from asthma are increasing in many countries. Of particular interest are reports describing rapid decompensation leading to respiratory arrest, sudden death or both in young patients with otherwise stable asthma. Risk factors for this include liability of the lower airways, lack of appreciation of the severity of airflow obstruction on the part of primary care physicians, and psychological factors, including emotional instability, depressive symptoms, and family dysfunction. The authors evaluated the occurrence of these episodes in relation to the peak outdoor mold sporulation season. Ten of the 11 patients with asthma who had respiratory arrest (91 percent) had positive skinpuncture tests for sensitivity to alternaria, as compared with 31 percent of the controls ( $P$ $<0.001$ ), and the serum lcvels of IgE antibodies to alternaria were elevated in all 9 patients tested. Exposure to the aeroallergen $A$. altemata is a risk factor for respiratory arrest in children and young adults with asthma. 